\title{
Status of the analysis for the search of polarization in the antiproton production process
}

D. Alfs ${ }^{1, \star}$, A. Asaturyan ${ }^{2}$, M. Carmignotto ${ }^{3}$, M. Diermaier ${ }^{4}$, W. Eyrich ${ }^{5}$, B. Głowacz ${ }^{1}$, D. Grzonka ${ }^{6}$, F. Hauenstein ${ }^{6}$, T. Horn ${ }^{3}$, K. Kilian ${ }^{6}$, S. Malbrunot-Ettenauer ${ }^{7}$, A. Mkrtchyan ${ }^{2}$, H. Mkrtchyan², P. Moskal ${ }^{1}$, P. Nadel-Turonski ${ }^{8}$, W. Oelert ${ }^{9}$, J. Ritman ${ }^{6}$, T. Sefzick ${ }^{6}$, V. Tadevosyan ${ }^{2}$, E. Widmann ${ }^{4}$, M. Wolke ${ }^{10}$, S. Zhamkochyan ${ }^{2}$, M. Zieliński ${ }^{1}$, A. Zink ${ }^{11}$, and J. Zmeskal ${ }^{4}$

${ }^{1}$ M. Smoluchowski Institute of Physics, Jagiellonian University, Kraków, Poland

${ }^{2}$ A.I. Alikhanyan Science Laboratory, Yerevan, Armenia

${ }^{3}$ Physics Department, The Catholic University of America, Washington DC, USA

${ }^{4}$ Stefan-Meyer-Institut für subatomare Physik, Wien, Austria

${ }^{5}$ Universität Erlangen, Erlangen, Germany

${ }^{6}$ Institut für Kernphysik, Forschungszentrum Jülich, Jülich, Germany

${ }^{7}$ Physics Department, CERN, Geneve, Switzerland

${ }^{8}$ Thomas Jefferson National Accelerator Facility, Newport News, Virginia, USA

${ }^{9}$ Johannes Gutenberg-Universität Mainz, Mainz, Germany

${ }^{10}$ Department of Physics and Astronomy, Uppsala University, Uppsala, Sweden

${ }^{11}$ Erlangen Centre for Astroparticle Physics (ECAP), Erlangen, Germany

\begin{abstract}
The P-349 experiment aims to test whether for antiprotons the production process itself can be a source of polarization in view of the preparation of a polarized antiproton beam. In this article we present the details of performed measurements and report on the status of the ongoing analysis.
\end{abstract}

\section{Motivation}

A wide range of fundamental effects in the hadronic sector can be investigated only by means of controlling the spin degrees of freedom. Although polarized proton beams and targets are produced routinely, the preparation of a polarized antiproton beam is still a challenge. A variety of methods to prepare such a beam were proposed [1], however most of them were already discarded due to the low degree of polarization or the expected small number of produced antiprotons.

The only experiment with polarized antiprotons was performed in FERMILAB where a proton beam of $800 \mathrm{GeV} / \mathrm{c}$ momentum produced $\bar{\Lambda}$ antihyperons. The observed mean polarization of antiprotons from the decay $\bar{\Lambda} \rightarrow \bar{p} \pi^{+}$was equal to $45 \%$. However, it is difficult to generate a well-defined beam by this method especially at lower energies [2].

Therefore, it would be desirable if antiprotons itself would have a non zero polarization degree when produced [3]. However, until now there were no experimental studies performed in this direction for antiprotons. The goal of the P-349 experiment is to test wether antiprotons are produced with polarization.

^e-mail: dominika.alfs@uj.edu.pl 


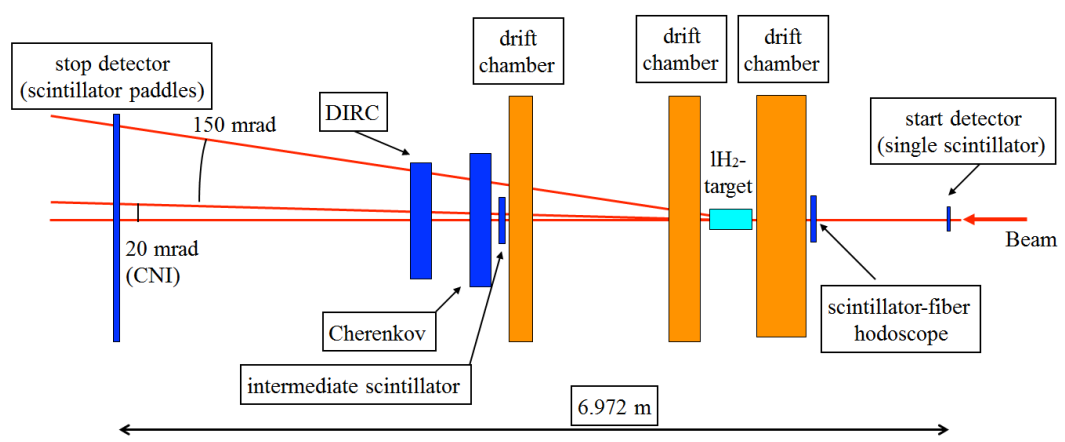

Figure 1. The scheme of the detector arrangement in the horizontal plane. The beam comes from the right side. The total angular range covered by the system is about $150 \mathrm{mrad}$ (horizontally as well as vertically) and the relevant scattering angle for the asymmetry measurement is about 20 mrad. Further explanation is given in the text.

\section{Analyzing reaction}

In order to measure the polarization of antiprotons the elastic $\bar{p} p$ scattering is used and the scattering asymmetry has to be determined in a region with known analyzing power. The Coulomb-Nuclear Interference (CNI) region is well suited for this kind of measurements because the analyzing power is well known and calculable at high energies. It was shown that in proton-proton and proton-antiproton elastic scattering the value is the same but with opposite sign. The maximum analyzing power is about $4.5 \%$ at a four momentum transfer equal to $t=-0.0037 \mathrm{GeV} / \mathrm{c}$ [4].

In the P-349 experiment the antiprotons have a momentum spectrum peaked around $3.5 \mathrm{GeV} / \mathrm{c}$ for which the high energy approximation is not valid anymore. However, according to preliminary calculations for lower antiproton momenta down to $5 \mathrm{GeV} / \mathrm{c}$, the maximum analyzing power is still about $4.5 \%$ for a four momentum transfer $t=-0.0025 \mathrm{GeV} / \mathrm{c}$ [5].

\section{Experimental setup}

The experiment was performed at the T11 beamline of the CERN/PS complex. Antiprotons were produced in the reaction $p p \rightarrow \bar{p} p p p$ by bombarding the solid target with an unpolarized proton beam of momenta equal to about $24 \mathrm{GeV} / \mathrm{c}$. This corresponds to the typical conditions of the antiproton beam production in the existent and planned facilities.

The system was operated in the air. It consisted of (see Fig. 1): scintillators for triggering purposes (start and stop) and TOF measurements (start, intermediate, stop), a scintillator fiber hodoscope for the beam profile control, a set of three drift chambers with vertical and inclined wires for precise $3 \mathrm{~d}$ track reconstruction [6], a Cherenkov detector for online pion background reduction (it served as trigger veto), and a DIRC for offline particle identification. The beam was scattered on a $15 \mathrm{~cm}$ long liquid hydrogen target.

\section{Particle identification and track finding}

In the first step of the analysis the ability of the online reduction of the pion background and the particle identification by the DIRC detector were verified. 


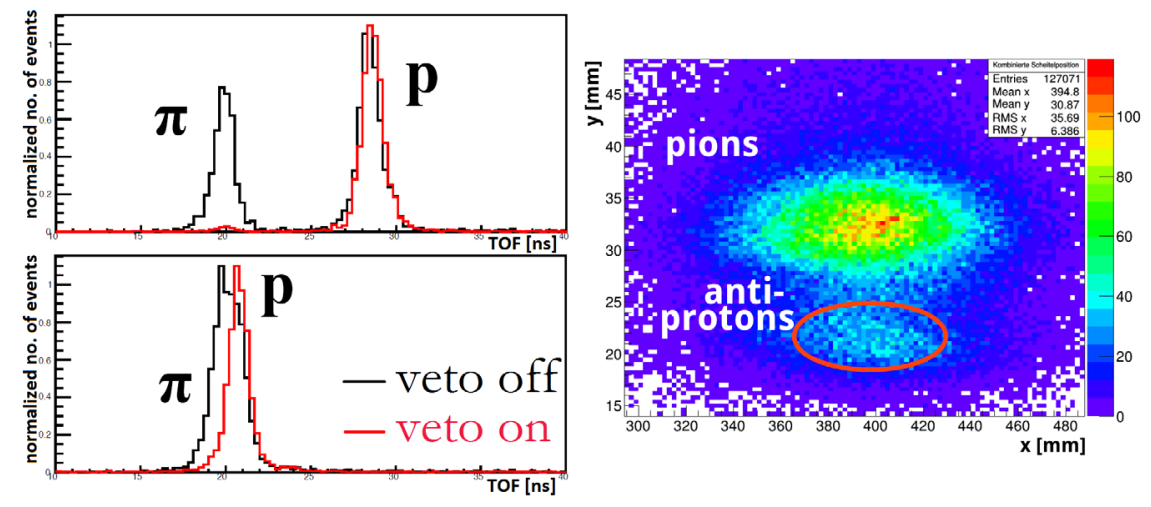

Figure 2. Left: Results of test measurements with the beamline set for positively charged particles of momenta $1 \mathrm{GeV} / \mathrm{c}$ (left upper part) and $3 \mathrm{GeV} / \mathrm{c}$ (left lower part). Right: Reconstruction of the Cherenkov arcs for each event in one spill in the measurement at $3.5 \mathrm{GeV} / \mathrm{c}$ with the bemline set to negatively charged particles. A clear separation of pions and antiprotons is visible.

The ability to suppress the pion background was confirmed in dedicated measurements with the beamline set to positively charged particles of different momenta. Results for momenta of $1 \mathrm{GeV} / \mathrm{c}$ and $3 \mathrm{GeV} / \mathrm{c}$ are presented in Fig. 2.

For the track finding the method of Hough transformation is applied which is often used in pattern recognition algorithms. In the P-349 experiment we use it to separate the track signals from background and electronic noise in the drift chambers. In the first step only the hits from vertical wires of the drift chambers are used. Each hit with coordinates $P(x, y)$ in 2-dimensional $X-Y$ space corresponds to the sinusoidal line $r=x \cos \theta+y \sin \theta$ in the Hough space (the space parametrized by $r$ and $\theta$ ). The intersection of the lines in the Hough space provides the parameters of the track candidate.

\section{Outlook}

The work on the calibration of the drift chambers and the track reconstruction is ongoing.

The majority of events are unscattered primary particles which pass trough the whole detector setup and are used to improve the drift chamber positioning and calibration. The next step is the extraction of antiproton scattering events and the reconstruction of their angular distribution.

This work was supported by the Polish Ministry of Science and Higher Education and by DAAD from resources of Bundesministerium für Bildung und Forschung (BMBF) and Marian Smoluchowski Kraków Research Consortium "Matter-Energy-Future" (KNOW).

\section{References}

[1] E. Steffens, AIP Conf. Proc. 1149, 80 (2009)

[2] A. Bravar et al., Phys. Rev. Lett. 77, 2626 (1996)

[3] K. Kilian et al., Int. J. Mod. Phys. A 26, 757 (2011)

[4] H. Okada et al., Phys. Lett. A 638, 450 (2006)

[5] D. Grzonka, et. al., Acta Phys. Polon. B 46, 191 (2015)

[6] J. Smyrski, et. al., Nucl. Instrum. Meth. A 541, 574 (2005) 